\title{
Design of Satellite Telemetry Based on PUS under Limited Downlink Channel
}

\author{
Yang LIU ${ }^{\mathrm{a}^{*}}$, Zongde LI, Xuejing DING ${ }^{\mathrm{b}}$ and Yuanyuan DAI \\ Shanghai Engineering Center for Microsatellites, Shanghai, China \\ a lyhit1980@163.com, ${ }^{b}$ happygirl_dxj@126.com
}

Keywords: Satellite Telemetry, PUS, Limited Downlink Channel.

Abstract. A design scheme of satellite telemetry based on PUS was introduced. Five types of packet were classified and the downloading strategy was elaborated to make full of the downlink resource under limited downlink channel. The application of it shows the feasibility and efficiency of it in satellite development.

\section{Introduction}

Packet telemetry is widely used in satellite downlink data exchange design now. To make the packet telemetry more universal and enhance the development and operation of space mission systems, Packet utilization standard (PUS) was brought forward by ECSS [1]. PUS defines the application-level interface between ground and space, which satisfies the requirements of electrical integration and testing and flight operations [2,3]. Though PUS is very convenient for satellite design, application of it is not easy when the downlink is limited, such as deep space projects. To transmit the telemetry exactly and effectively, different PUS service and subservice should be tailored and selected. A design of PUS application was elaborated in this article on how to design and adjust telemetry under limited downlink channel.

\section{Packet Structure}

Telemetry packets conform to the structure defined and shown in Fig. 1 [1].The different segment definition is listed as bellowed.

Version number: The edition of telemetry packet structure.

Type: The sign distinguishes between telecommand packets and telemetry packets. For telemetry packets, the type $=0$.

Data Field Header Flag: Indicates the presence or absence of a data field header. With the exception of the spacecraft time source packet, all telemetry source packets shall have a data field header, for which this bit shall be set to 1 .

Application Process ID (APID): Corresponds uniquely to an on-board application which is the source of this packet. The choice of Application Process ID values is mission-specific. APID $=0$ is reserved for the time packet. APID = "11111111111" (eleven ones) is reserved for idle packets.

Grouping Flags: The grouping flags shall be used when a number of telemetry source packets originating from the same application process are sent in a group. All standard telemetry source packets defined within PUS are stand-alone packets.

Source Sequence Count: A separate source sequence count shall be maintained by each APID and shall be incremented by 1 whenever it releases a packet. If the application process can send distinct packets to distinct destinations using the optional Destination ID field shown below, then a separate source sequence count is maintained for each destination. Therefore the counter corresponds to the order of release of packets by the source and enables the destination to detect missing packets.

Packet Length: The packet length field specifies the number of octets contained within the packet data field. 
Spare: To maintain symmetry with the telecommand packet data field header, this bit shall be reserved and set to zero.

TM Source Packet PUS Version Number: By changing the PUS version number, future variations of the telemetry source packet can be introduced.

Service Type: This indicates the service to which this telemetry source packet relates. Service types 0 to 127 shall be reserved for PUS, service types 128 to 255 are mission-specific.

Service Subtype: Together with the service type, the subtype uniquely identifies the nature of the service report constituted by this telemetry source packet. Within standard services, subtypes 0 to 127 shall be reserved for PUS, subtypes 128 to 255 are mission-specific. Within mission-specific services, all subtypes ( 0 to 255 ) are available for mission-specific use. The definition of service type and subtype shall be unique across all application processes and the combination of these fields can be used on the ground to determine the processing priority level.

Destination ID: This field identifies the destination of the telemetry source packet. The length of this field is known implicitly for the mission. This field shall be systematically omitted if the mission has only a single destination for telemetry source packets or has no requirement to

\begin{tabular}{|c|c|c|c|c|c|c|c|c|}
\hline \multicolumn{7}{|c|}{ Packet Header } & \multicolumn{2}{|c|}{ Packet Data Field } \\
\hline \multicolumn{4}{|c|}{ Packet ID } & \multicolumn{2}{|c|}{ Packet Sequence Control } & \multirow{3}{*}{$\begin{array}{l}\text { Packet } \\
\text { Length }\end{array}$} & \multirow{3}{*}{$\begin{array}{c}\text { Data Field } \\
\text { Header }\end{array}$} & \multirow{3}{*}{$\begin{array}{c}\text { Source } \\
\text { Data }\end{array}$} \\
\hline $\begin{array}{l}\text { Version } \\
\text { Number }\end{array}$ & Type & $\begin{array}{l}\text { Data Field } \\
\text { Header Flag }\end{array}$ & APID & $\begin{array}{l}\text { Grouping } \\
\text { Flags }\end{array}$ & $\begin{array}{c}\text { Source } \\
\text { Sequence } \\
\text { Count }\end{array}$ & & & \\
\hline 3 & 1 & 1 & 11 & 2 & 14 & & & \\
\hline \multicolumn{4}{|c|}{16} & \multicolumn{2}{|c|}{16} & 16 & Variable & Variable \\
\hline
\end{tabular}

\begin{tabular}{|c|c|c|c|c|c|c|}
\hline Spare & $\begin{array}{c}\text { TM Source } \\
\text { Packet PUS } \\
\text { Version } \\
\text { Number }\end{array}$ & Spare & $\begin{array}{c}\text { Service } \\
\text { Type }\end{array}$ & $\begin{array}{c}\text { Service } \\
\text { Subtype }\end{array}$ & $\begin{array}{c}\text { Destination } \\
\text { ID }\end{array}$ & Time \\
\hline 1 & 3 & 4 & 8 & 8 & 8 & 56 \\
\hline
\end{tabular}

Fig. 1 PUS telemetry structure

distinguish between destinations.

Time: The on-board reference time of the packet, expressed in either the CUC or CDS format.

\section{Packet Definition with PUS}

To fully and efficiently utilize the limited downlink resource, the downloading parameters must be selected and classified strictly. The parameters should be transmitted according to the usage requirements. Based on the strategy brought forward in this paper, parameters are divided into five types according to their importance. Then these parameters are packed in different packets. These five type packets are key packet, important packet, state packet, parameter packet and automatic packet. The characters of these packets are listed as follows.

Table 1 Packet classification

\begin{tabular}{cccc}
\hline Type & $\begin{array}{c}\text { Transmission } \\
\text { condition }\end{array}$ & Transmission mode & Stop condition \\
\hline Key packet & Always & Periodic & Never \\
Important packet & Judged by OBDH & Periodic & Judged by ground \\
State packet & Judged by ground & Periodic & Judged by ground \\
Parameter packet & $\begin{array}{c}\text { Judged by ground } \\
\text { Judged by OBDH or } \\
\text { Automatic packet }\end{array}$ & Asynchronous & Automatic by OBDH \\
\hline
\end{tabular}




\section{Key Packet}

The key packets include the most critical parameters for the satellites, such as primary power voltage. The key packets should be transmitted with high frequency and never be suspended. The key packets always take up the downlink channel with high speed, so the parameters must be selected carefully to avoid holding too much resource under limited downlink.

The key packets can be transmitted directly by PUS service 3-"Housekeeping and diagnostic data reporting".

\section{Important Packet}

The important packets contain the important parameters for the satellites healthy state. These parameters are usually regular or steady, so they do not need to be transmitted continually. The health state value of the parameters will be watched by the OBDH. When the satellites are not on a healthy state, the OBDH will transmit the packet to the ground automatically and cyclically to inform the abnormal of the satellite. When the problems are solved, the important packet will be stopped by the ground TC to reduce the usage of limited downlink.

To implement the important packets conveniently and simply, service type 5 is chosen and three mission-specific service subtypes (ST) are defined.

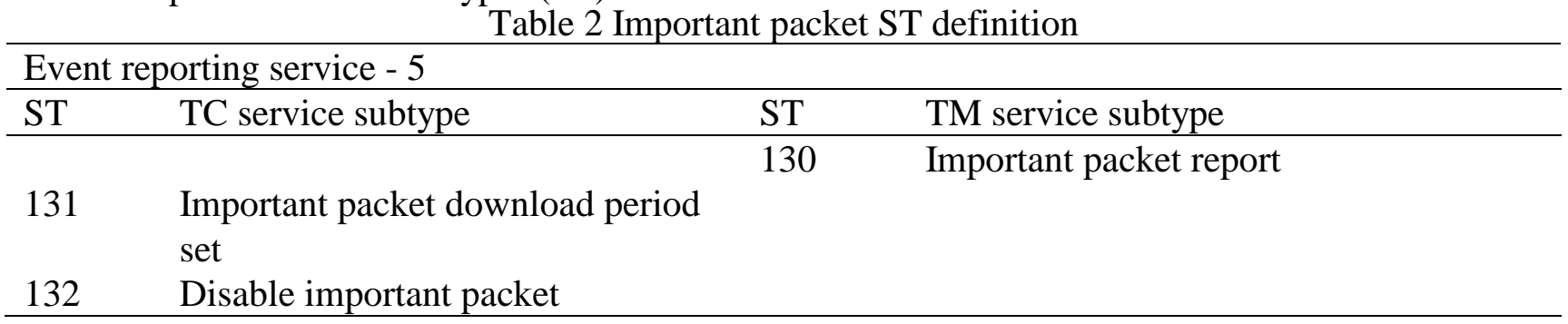

\section{Definition of $S T(5,130)$}

\begin{tabular}{|c|c|c|c|}
\hline $\mathrm{N}$ & IPID & IPPID & Parameters \\
\hline $\begin{array}{c}\text { Unsigned } \\
\text { integer }\end{array}$ & Enumerated & Enumerated & Any \\
\hline
\end{tabular}

$\mathrm{N}$ : the number of parameters downloaded in important packet.

IPID: the identification of important packet

IPPID: the identification of parameter in important packet.

Parameters: the value of downloading parameters.

\section{Definition of ST $(5,131)$}

\begin{tabular}{|c|c|}
\hline IPID & Period \\
\hline Enumerated & $\begin{array}{c}\text { Unsigned } \\
\text { integer }\end{array}$ \\
\hline
\end{tabular}

IPID: the identification of important packet

Period: the interval of important packet download is defined. The actual time value is equal to 2 period.

\section{Definition of $S T(5,132)$}

IPID: the identification of important packet 


\section{State Packet}

The state packets consist of the assistant state parameters of the satellites. It will not be downloaded normally for saving the downlink resource. When the ground needs more information to diagnose the problem or observe the satellite state more precisely, the ground will send the telecommands and the corresponding important packets will be downloaded cyclically. It also can be prevented by the ground TC.

The state packets can be achieved directly by PUS service 3-"Housekeeping and diagnostic data reporting”. To make it more clearly, four more mission-specific service subtypes are defined.

Table 3 State packet ST definition

\begin{tabular}{llcl}
\hline \multicolumn{4}{l}{ Housekeeping and diagnostic data reporting service - 3 } \\
\hline ST & TC service subtype & ST & TM service subtype \\
\hline 129 & Enable state packet & 130 & State packet report \\
& & & \\
131 & State packet download speed set & & \\
132 & Disable state packet & & \\
\hline
\end{tabular}

\section{Definition of $S T(3,129)$}

SPID: the identification of state packet

\section{Definition of ST $(3,130)$}

\begin{tabular}{|c|c|c|c|c|}
\hline $\mathrm{N}$ & SPID & Parameter 1 & $\ldots .$. & Parameter N \\
\hline $\begin{array}{c}\text { Unsigned } \\
\text { integer }\end{array}$ & Enumerated & Any & $\ldots . .$. & Any \\
\hline
\end{tabular}

$\mathrm{N}$ : the number of parameters downloaded in state packet.

SPID: the identification of state packet.

Parameter 1 Parameter $\mathrm{N}$ : the value of downloading parameters.

Definition of $S T(3,131)$

IPID: the identification of state packet

\begin{tabular}{|c|c|}
\hline SPID & Period \\
\hline Enumerated & $\begin{array}{c}\text { Unsigned } \\
\text { integer }\end{array}$ \\
\hline
\end{tabular}

Period: the interval of state packet download is defined. The actual time value is equal to $2^{\text {period }}$.

\section{Definition of $S T(3,132)$}

SPID: the identification of state packet.

\section{Parameter Packet}

The parameter packets comprise the configuration parameters of the system or equipment. It only is downloading when the ground send TC to update the parameters or check the value of the parameters. The packets are transmitted asynchronously.

The parameter packets can be carried out by PUS service 4-"Parameter statistics reporting". To make it more flexible, three mission-specific service subtypes are defined. 
Table 4 Parameter packet ST definition

\begin{tabular}{llll}
\hline \multicolumn{2}{l}{ Parameter statistics reporting service -4} & & \\
\hline ST & TC service subtype & ST & TM service subtype \\
\hline 129 & Enable parameter packet & 130 & Parameter packet report \\
& & & \\
131 & Number of parameter packet set & & \\
\hline
\end{tabular}

\section{Definition of $S T(4,129)$}

IPID: The identification of parameter packet

Definition of $S T(4,130)$

\begin{tabular}{|c|c|c|c|c|}
\hline $\mathrm{N}$ & PPID & Parameter 1 & $\ldots \ldots$ & Parameter $\mathrm{N}$ \\
\hline $\begin{array}{c}\text { Unsigned } \\
\text { integer }\end{array}$ & Enumerated & Any & $\ldots \ldots$ & Any \\
\hline
\end{tabular}

$\mathrm{N}$ : The number of parameters downloaded in parameter packet.

SPID: The identification of parameter packet

Parameter 1 Parameter N: The value of downloading parameters.

\section{Definition of $S T(4,131)$}

\begin{tabular}{|c|c|}
\hline \multicolumn{1}{|c|}{ PPID } & NUM \\
\hline Enumerated & $\begin{array}{c}\text { Unsigned } \\
\text { integer }\end{array}$ \\
\hline
\end{tabular}

IPID: The identification of parameter packet

NUM: The number of parameter packet to be downloaded.

\section{Automatic Packet}

There are totally 52 standard PUS subservices for telemetry defined by PUS. The basic telemetry subservices are listed in table 2. Most of the telemetry packets are automatic packets and respond to the related telecommand subservices.

Table 5 Automatic packet ST definition

\begin{tabular}{ccl}
\hline Service & Subservice & Service content \\
\hline 1 & 1 & Telecommand Acceptance Report -- Success \\
& 2 & Telecommand Acceptance Report -- Failure \\
3 & 25 & Housekeeping Parameter Report \\
& 26 & Diagnostic Parameter Report \\
& 1 & Normal/Progress Report \\
5 & 2 & Error/Anomaly Report -- Low Severity \\
& 3 & Error/Anomaly Report -- Medium Severity \\
9 & 4 & Error/Anomaly Report -- High Severity \\
12 & 2 & Time Report \\
& 12 & Check Transition Report \\
& 1 & First Downlink Part Report \\
13 & 2 & Intermediate Downlink Part Report \\
& 3 & Last Downlink Part Report \\
& 4 & Downlink Abort Report \\
& 14 & Uplink Reception Acknowledgement Report \\
& 16 & Reception Abort Report \\
& 2 & Connection Test Report \\
\hline
\end{tabular}




\section{Telemetry Download Strategy}

To classify and separate the different packets more clearly, Virtual Channel Identifier(VCID) values are also defined to allow to segregate the following TM flows.

- $\quad 000 \mathrm{H}$ : Key packets

- $001 \mathrm{H}:$ Important packets

- $\quad 010 \mathrm{H}:$ State packets

- $011 \mathrm{H}:$ Parameter packets

- $100 \mathrm{H}:$ Automatic packets

- $101 \mathrm{H}$ : Idle

Through this classification, only the key packets are transmitted in normal circumstances. Though the key packets are downloaded with high frequency, they only occupy a very limited telemetry channel resource.

When there are some abnormalities of satellites, the corresponding important packets will be transmitted under the control of OBDH. For more details, the ground can send TCs to get the state packets and parameter packets. Since parameter packets are asynchronous, they will spend little channel resource. For important packets and state packets, their download frequencies can be regulated by ground TCs to find more details. Under this condition, the transmission period of some key packets which not need to be observed at the same time can be increased to save more downlink resource. Thus the telemetry is used effectively and efficiently under limited downlink channel.

All the telemetries are defined with PUS services (standard and mission-specific). Most of them can be inherited and used by the other projects directly. If there are some new requirements, it can be realized by the new service subtype with very little change and new definition.

\section{Conclusion}

PUS is applied in satellite telemetry packet design and classification under limited downlink channel resource. The usage of PUS not only helps to make the telemetry much more standard and universal, but also helps to make full of use the downlink resource. The application of this design has been verified in some satellite project and the result shows that it is a good exploration for satellite development.

\section{Acknowledgements}

The project was supported by the National High Technology Research and Development Program of China (863 program, Grant No.2011AA12A101).

\section{References}

[1] Ground systems and operations Telemetry and telecommand packet utilization [S]. Netherlands, ECSS-E-70-41A, January 30, 2003.

[2] Valera S, Melton B, Parkes A. Status of ECSS standards for ground systems and operations [C]. ESA EGSE Workshop, ESA ESTEC Noordwijk, Netherlands, February 11-12, 2003.

[3] Merri M, Melton B, Valera S. The ECSS packet utilization standard and its support tool [C]. ESA Space Operations Workshop, Houston, USA, October 9-12, 2002. 